\title{
A New Approach to Measure Social Capital using Game-Theoretic Techniques
}

TOMASZ P. MICHALAK

University of Oxford, UK, and University of Warsaw, Poland

TALAL RAHWAN

Masdar Institute of Science and Technology, UAE

STEFANO MORETTI

CNRS and Paris-Dauphine University, France

RAMASURI NARAYANAM

IBM Research, India

OSKAR SKIBSKI

Kyushu University, Japan

PIOTR SZCZEPAŃSKI

Warsaw University of Technology and Hewlett-Packard GD Poland, Poland

MICHAEL WOOLDRIDGE

University of Oxford, UK

\begin{abstract}
Although the notion of social capital has been extensively studied in various bodies of the literature, there is no universally accepted definition or measure of this concept. In this article, we discuss a new approach for measuring social capital which builds upon cooperative game theory. The new approach not only turns out to be a natural tool for modeling social capital, but also captures various aspects of this phenomenon that are not captured by other approaches.

Categories and Subject Descriptors: J.4 [Social and Behavioral Sciences]: Economics

General Terms: Social capital, Social computing, Cooperative game theory

Additional Key Words and Phrases: Social capital, Shapley value, network centrality
\end{abstract}

\section{INTRODUCTION}

Social capital is one of the fundamental concepts in sociology [Coleman 1990]. Intuitively, we can think of social capital as the ability of individuals to profit from their position in the society, i.e., from their connections in the social network. Although it has been extensively studied, there is no single definition or measure that captures all facets of this concept. In this article, we comment on recent works that build upon cooperative game theory to develop advanced measures of social capital. According to Borgatti et al. [1998], social capital is understood in the literature in two conceptually different ways, called group and individual social capital. The former interprets social capital as the quality or performance of a given group of individuals in a social network [Putnam 1995]. The latter interprets social capital as the value of an individual's social connections, which are seen as potential

The corresponding author's address: tomasz.michalak@cs.ox.ac.uk 
sources of information, power, or opportunities [Burt 1992]. Moreover, Borgatti et al. argue that the analysis of individual social capital can be done externally, while for group social capital it can be done either internally and externally. For instance, the internal analysis of group social capital would consider any given group as a universe that consists of connections, norms and trust, but nothing outside the group is modeled. In contrast, external analysis of group social capital would focus on how the members of the group are connected to the outside world. Since social capital depends on how the different actors are positioned in their social network, existing measures of social capital are usually built upon methods pertaining to social network analysis [Borgatti et al. 1998]. The following are a few such measures, categorized according to the type of social capital they capture:

-(A): Individual-centric, External Measures - those focus on the connections of an individual. Some key examples include the ego-network measures [Burt and Minor 1983], the structural hole measures [Burt 1992], and the standard centrality measures such as closeness, betweenness, and eigenvector centrality.

-(B): Group-centric, Internal Measures - those focus on internal connections of a group and are often referred to as internal measures for collective actors. They include the average or maximum distance and homophily [Borgatti et al. 1998].

-(C): Group-centric, External Measures - those focus on internal connections of a group a are often to as external measures for collective actors. Among the most important examples are group-degree (i.e., the number of the group's neighbors), group-closeness, and group-betweenness (defined similarly) [Everett and Borgatti 1999].

Although the above measures are able to capture some aspects of social capital, they cannot capture others. Below are two particular deficiencies that we are especially interested in:

(i) While existing measures quantify each of the above types of social capital separately, none of them sheds light on the interactions between those types;

(ii) Since the aforementioned measures originate from social network analysis, they are limited to the aspects of social capital related solely to network topology.

In this article, we consider a more advanced approach to the measurement of social capital, which builds upon recent literature that uses concepts rooted in cooperative game theory to analyze social networks. We argue that this new approach is able to address both problems (i) and (ii) outlined above.

In the next section, we present the theoretical underpinnings of this approach.

\section{GAME-THEORETIC CENTRALITY}

In a cooperative game, players are allowed to form coalitions and share the payoff generated by cooperation. Formally, given a set of players $N$, the payoff generated by a coalition $C \subseteq N$ is denoted by $\nu(C)$. There exist well-established schemes to divide the payoff attainable by all the players working together, $\nu(N)$. The most prominent one is the Shapley value [Shapley 1953], the theoretical properties of which have been extensively studied for decades. To define the Shapley value, let us denote by $\operatorname{MC}(C, i)$ the marginal contribution of player $i$ to coalition $C$, i.e., 
$\operatorname{MC}(C, i)=\nu(C \cup\{i\})-\nu(C)$. Then, the payoff of $i$ according to the Shapley value is:

$$
S V_{i}(\nu)=\sum_{C \subseteq N \backslash\{i\}} \frac{|C| !(|N|-|C|-1) !}{|N| !} M C(C, i) .
$$

Game-theoretic centrality borrows the above apparatus and applies it to quantify the role played by nodes in a network. To this end, the nodes are considered to be players in a cooperative game, whereby the payoff of any coalition of nodes is assumed to capture in some way the performance of these nodes in the network. By drawing this analogy, the game-theoretic payoff division schemes that evaluate the role (also interpreted as power) of a player in a coalitional game can be used to evaluate the role of nodes in the network. As an example of such an approach, Keinan et al. [2004] divided an animal's brain into several interconnected sections that form a network. After that, certain groups of nodes (i.e., sections of the brain) were temporarily lesioned, and a payoff was assigned to each such group based on how it influenced the animal's performance. Finally, the Shapley value was used to rank the role played by individual nodes.

Game-theoretic centrality has been advocated in various domains as a networkanalysis tool, delivering insights unattainable by classical measures. In this article, we argue that this tool naturally captures various aspects of social capital. However, its use poses significant computational challenges inherited from coalitional games. In particular, given a network with a set of nodes, $V$, and a set of edges, $E$, there are $2^{|V|}$ coalitions to consider. If each of them has to be considered separately then the exact value of a game-theoretic centrality can only be computed for very small networks. Interestingly, however, it has been recently shown that certain classes of game-theoretic centrality are computable in polynomial time [Michalak et al. 2013; Szczepański et al. 2012]. We will discuss one such class in the next section.

\section{THE OWEN VALUE-BASED MEASURE OF SOCIAL CAPITAL}

The Owen value [Owen 1977] is an extension of the Shapley value to situations in which the players are already divided into coalitions (rather than situations where players are contemplating which coalitions to form). The relevance to our socialnetwork context becomes apparent as soon as we think of those already-formed coalitions as communities. Similarly to the Shapley value, the Owen value quantifies the role of a player, but now taking into account the role of the community that this player belongs to. Thus, a player may be weak by herself, but if she belongs to a strong community, then her role will grow stronger according to the Owen value.

To define the Owen value given the set of communities $M=\left\{C_{1}, \ldots, C_{m}\right\}$, let us first introduce the concept of the quotient game whereby the set of players is $M$, (i.e., the players are the communities themselves), and the payoff of a coalition of players (i.e., a union of communities), $R \subseteq M$ is: $\nu^{Q}(R)=\nu(\cup R)$. We are now ready to define the Owen value of player $i \in C_{j} \in M$. This is:

$$
O V_{i}(\nu, M)=\sum_{R \subseteq M \backslash\left\{C_{j}\right\}} \frac{|R| !(|M|-|R|-1) !}{|M| !} \sum_{C \subseteq C_{j} \backslash\{i\}} \frac{|C| !\left(\left|C_{j}\right|-|C|-1\right) !}{\left|C_{j}\right| !} \operatorname{MC}(R \cup C, i) .
$$

Intuitively, the computation of the Owen value can be thought of as a two-step 
process. In the first step, communities play the game between themselves and receive their respective Shapley values. In the second step, the values of these communities are, in turn, divided among their members, again, in spirit of the Shapley value.

Recently, Szczepański et al. [2014] proposed the game-theoretic extension of degree centrality based on the Owen value. Specifically, given a community structure $M$, this centrality is computed for each node $v \in V$ as $O V_{v}(\nu, M)$, where $\nu(C)=\mid$ Neighbors $(C) \mid$. It has an interesting interpretation as a measure of social capital. In particular, the social capital of an individual is increased (decreased) if she belongs to a community that is rich (poor) in terms of social capital. For instance, if a lawyer is not particularly well connected (i.e., has low degree), her social capital is still increased by the fact that she belongs to the well-connected community of lawyers. As such, this is the first social capital measure that evaluates individual actors both in the context of their relationship with the external world as well as the context of the external role of their community, thus reflecting types (A) and (C) of social capital (see the introduction).

Interestingly, while the Owen value is computationally challenging, Szczepański et al. [2014] showed that their measure can be computed in just $O(|V|+|E|)$ time, which makes this concept practical even for large networks.

\section{SOCIAL CAPITAL AND THE MYERSON VALUE}

Having presented a measure of social capital based on the Owen value, we now present yet another measure, based on the Myerson value. Generally speaking, the Myerson value is a solution concept from cooperative game theory, developed by Myerson [1977] as an extension of the Shapley value to settings where cooperation is restricted via a communication graph: only if players are connected, either directly via an edge or indirectly via intermediaries, can they effectively cooperate. Specifically, the Myerson value of player $i$ is:

$$
M V_{i}(\nu, G)=S V_{i}\left(\nu^{G}\right),
$$

where $\nu^{G}$ specifies the payoffs of coalitions as follows: if coalition $C$ is connected in $G$ then $\nu^{G}(C)=\nu(C)$. Otherwise, if $C$ is a disconnected coalition made of $m$ components, $K_{1}, \ldots, K_{m}$, then $\nu^{G}(C)=\sum_{K_{i}} v\left(K_{i}\right)$.

The Myerson value has a number of desirable properties, e.g., it is the only solution concept satisfying the following two intuitive axioms: (i) "efficiency" - the entire payoff of a connected coalition is divided among its members; (ii) "fairness" the payoffs of two players should increase (decrease) by exactly the same amount when the edge between them is added (deleted) from the communication graph.

Among the various applications of the Myerson value, Moretti et al. [2010] used it to evaluate the relevance of genes in biological networks. Here, players correspond to genes, edges correspond to interaction ties, and coalitions of genes are evaluated based on the overall magnitude of the interaction between those genes and an $a$ priori given set of key-genes (e.g., a set of genes that are involved in a certain biological condition of interest).

González-Arangüena et al [2011] proposed a dedicated measure of social capital that builds upon the Myerson value. In particular, the authors argue that if the communication graph was complete (i.e., if every two individuals in the society 
knew each other) then such a situation can be modeled as a standard coalitional game, and the role of an individual can be measured using the Shapley value. On the other hand, since in reality the communication graph is incomplete (i.e., one is typically not acquainted with every other member of the society), the role of an individual can be measured using the Myerson value. Based on this argument, the authors propose to measure social capital as the difference between one's role in the actual social network, and her role in a hypothetical, complete network. That is, social capital is the impact of the social-network topology on the role that an individual plays in the society.

With the above approach, the authors measure an individual's social capital while considering both the internal and external social capital of her community, thus reflecting types (A), (B) and (C) of social capital (see the introduction). More specifically, the "efficiency" axiom of the Myerson value takes into account the internal role of the community, by protecting the internal capital of the community from any potential changes that may occur outside of it. On the other hand, the "fairness" axiom implies that the members of the community are influenced by their connections to non-members, which reflects the external capital.

While it is computationally challenging to computer the Myerson value exactly [Michalak et al. 2013; Skibski et al. 2014], approximation techniques such as Monte Carlo sampling have been shown to be effective (see, e.g., [Narayanam et al. 2014] and [Moretti 2014]).

\section{CONCLUSIONS AND FURTHER DIRECTIONS}

We discussed a new approach for measuring social capital, based on techniques pertaining to cooperative game theory. We argued that the new measures are able to capture aspects of social capital that are unattainable by previous approaches.

Inspired by the ever-increasing availability of data about social interactions, an interesting direction for future research is to expand the repository of games based on which tractable social-capital measures can be defined. Furthermore, empirical evaluations of these measures, coupled with the new insights brought forward by the game-theoretic approach, may well expand on our understanding of social capital, and social substance in general.

Acknowledgements: We thank the Editor, Shaddin Dughmi, for various comments and suggestions that helped improve this article. Oskar Skibski was supported by JSPS KAKENHI Grant (24220003). Tomasz Michalak and Michael Wooldridge were supported by the European Grant (24220003). Tomasz Michalak and Michael Wooldridge were supported by the European
Research Council under Advanced Grant 291528 ("RACE"). Oskar Skibski, Talal Rahwan, Piotr Szczepański, and Tomasz Michalak were supported by the Polish National Science Center grant DEC-2013/09/D/ST6/03920.

\section{REFERENCES}

Borgatti, S., Jones, C., And Everett, M. 1998. Network Measures of Social Capital. Connections 21, 2, 27-36.

Burt, R. And Minor, M. 1983. Applied network analysis: a methodological introduction. Sage Publications.

Burt, R. S. 1992. Structural holes: The social structure of competition. Harvard University Press.

Coleman, J. 1990. Foundations of Social Theory. Harvard University Press, Cambridge MA.

E. GonzÁlez-Aranguena, A.B. Khmelnitskaya, C. M. And del Pozo, M. 2011. A social capital index. In In Numerical analysis and applied mathematics ICNAAM,. 2041-2043. 
Everett, M. G. And Borgatti, S. P. 1999. The centrality of groups and classes . Journal of Mathematical Sociology 23, 3, 181-201.

Keinan, A., Hilgetag, C. C., Meilijson, I., And Ruppin, E. 2004. Casual localization of neural function: the shapley value method. Neurocomputing 58-60, 0, 215-222.

Michalak, P. T., Aaditha, K. V., Szczepanski, P. L., Ravindran, B., and Jennings, N. R. 2013. Efficient computation of the shapley value for game-theoretic network centrality. Journal of Artificial Intelligence Research 46, 607-650.

Michalak, T. P., Rahwan, T., Szczepanski, P. L., Skibski, O., Narayanam, R., Wooldridge, M. J., And Jennings, N. R. 2013. Computational analysis of connectivity games with applications to the investigation of terrorist networks. IJCAI'13.

Moretti, S. 2014. On the role of coalitional network games in modelling the social dimension in ecosystem management. Strategic Behavior and the Environment 4, 2, 155-186.

Moretti, S., Fragnelli, V., Patrone, F., and Bonassi, S. 2010. Using coalitional games on biological networks to measure centrality and power of genes. Bioinformatics 26, 21, 2721-2730.

Myerson, R. 1977. Graphs and cooperation in games. Mathematics of Operations Research 2, 3, $225-229$.

Narayanam, R., Skibski, O., Lamba, H., and Michalak, T. 2014. A shapley value-based approach to determine gatekeepers in social networks with applications. In ECAI'14.

Owen, G. 1977. Values of games with a priori unions. In Mathematical economics and game theory: Essays in honor of Oskar Morgenstern. Lecture Notes in Economics and Mathematical Systems, vol. 141. Springer, 76-88.

Putnam, R. D. 1995. Bowling alone: America's declining social capital. Journal of Democracy 6, 65-78.

Shapley, L. S. 1953. A value for n-person games. In In Contributions to the Theory of Games, volume II, H. Kuhn and A. Tucker, Eds. Princeton University Press, 307-317.

Skibski, O., Michalak, T., Rahwan, T., And Wooldridge, M. 2014. Algorithms for the shapley and myerson values in graph-restricted games. In $A A M A S$ '14.

Szczepanski, P., Michalak, T., And Wooldridge, M. 2014. A centrality measure for networks with community structure based on a generalization of the owen value. In ECAI'14.

Szczepański, P. L., Michalak, T., and Rahwan, T. 2012. A new approach to betweenness centrality based on the shapley value. In $A A M A S^{\prime} 12$. 